\title{
Noise Radar Technology: \\ Pseudorandom Waveforms and their Information Rate
}

\author{
Gaspare Galati, Gabriele Pavan, Francesco De Palo \\ Tor Vergata University - Department of Electronic Engineering, "Vito Volterra" Centre and CNIT \\ Via del Politecnico, 1-00133 Rome, ITALY \\ gaspare.galati@uniroma2.it, gabriele.pavan@uniroma2.it, francesco.de.palo@uniroma2.it
}

\begin{abstract}
The well-known LPI (Low Probability of Intercept) and antispoofing capabilities of some radar waveforms as well some other desirable feature such as a low sidelobes level of their autocorrelation function can be enhanced by "tailored" pseudorandom sequences, whose phase is made up by a deterministic part plus a variable random term. With such an approach it is possible to design a virtually unlimited number of noisy waveforms with good auto-correlation properties (PSLR, i.e. Peak to Side Lobe Ratio) and, for MIMO applications, good orthogonality between them. In this paper the LPI characteristic are analyzed by evaluation of the variation of their information rate with their "randomness" varying. It results that an ad-hoc tradeoff between the different requirements (LPI, PSLR) is required in most cases.
\end{abstract}

Keywords - Entropy, entropy rate, mutual information, noise waveform radar, noise radar technology.

\section{INTRODUCTION}

Modern Electronic Warfare (EW) systems are aimed to deny the radar function of the enemy by a sequence of actions starting from the acquisition and the off-line analysis of radar signals (Electronic Intelligence, ELINT) and going on with the real-time detection and interception/analysis using ESM (Electronic Support Measures) systems with the aim to optimize radar jamming strategies and, whenever possible, to perform a Specific Emitter Identification. Short, high peak power pulses such as the ones from legacy magnetron-based radar are relatively easy to detect, identify and measure, but today complicated waveforms are being more and more used to improve some significant properties of radars and increase it performance, including a renewed interest in the old concept of continuous wave radar, an architecture often used nowadays. The more and more frequent usage of sophisticated waveforms in pulse-compression radar and of low-power signals (order of $\mathrm{W}$ versus the tens or hundreds of $\mathrm{kW}$ from magnetrons) in Continuous Wave radar is a challenge to the EW engineers [1]. A group of very interesting waveforms is based on bandlimited noise [2], sometimes modified, or "tailored", to obtain some required properties for the ambiguity function, in the frame of the so-called Noise Radar Technology (NRT) [3], [4]. Noise radar technology (NRT) poses a new, or additional, threat due to the unpredictability of radar signals themselves, even when the operational scenario allows ELINT or ESM receivers to work with a very large signal-to-noise ratio.
In the NRT community the question of "the amount of needed randomness" for the radiated waveforms is still open. Some expert would call for a "pure (and analog) noise" as originated by a noisy physical system (an amplifier, a Zener diode, a chaotic circuit and so on) while other experts prefer modern, all-digital NRT architectures using sequences from a Pseudo Random Numbers (PRN) generator. The objection that every pseudorandom sequence has a repetition period that can impair the LPI is easily rejected when considering that modern, open-access PRN's [5] can reach periods as long as $2^{19937}$ (Mersenne Twister algorithm) [6], i.e. can generate "new" pseudorandom samples at $100 \mathrm{MHz}$ (or better, Msps) frequency for $1.36 \cdot 10^{5994}$ years, a rather long time when compared to the estimated age of the universe, i.e. $1.37 \cdot 10^{10}$ years.

However, a "pure digital noise”, e.g. a Gaussian sequence with some suitable spectral density in the radar band, is not always a good choice for NRT systems. The problems are two. First, most radar transmitters operate in saturation, i.e. with constant envelope during the emission (i.e. the waveform duration); the penalty of a possible linear emission would be as much as $10-11 \mathrm{~dB}$ in the radar power budget, unacceptable for medium and long range applications [7]. Second, in Noise Radar the correlation processing of echoes (duration $\mathrm{T}$, bandwidth B) generates sidelobes (better, random fluctuations) whose suppression is difficult and whose peak amplitude below the maximum of the compressed signal is of the order $K-10 \cdot \log _{10}(B T)$ decibels, with $K$ close to $11-12 \mathrm{~dB}$. Hence the interest in "tailored" pseudorandom waveforms [8] with $(i)$ a crest factor equal to, or close to, the unit (for maximum exploitation of the transmitter power) and (ii) fairly low sidelobes in Range and Doppler. Of course these waveforms must significantly differ from realizations of band pass, Gaussian noise. Hence, question arises about their "degree of randomness". In practice, the main question is about their properties in the ELINT - ESM context, and can be reworded as follows: how much information about type and parameters of the signals emitted from particular radar (or a class of radar) is obtained by analyzing more and more samples from the radar emission? Probably, there is not a unique answer, independent of the operation and performance of the ELINT - ESM and of the operational theatre, but a rather general answer may be searched in terms of Information Theory, where a measure of information contained in a signal is related to the entropy concept [9]. This is fundamental in many signal processing applications such as source coding, bit-rate reduction or data 
compression, where the number of bits is reduced by identifying and eliminating statistical redundancy or unnecessary information and removing it.

In NRT pseudo-random signals are transmitted and correlation processing of echoes is used with the primary goal of LPI capability. To generate a pseudo-noise waveform for NRT applications we may consider a sequence of time ordered samples obtained by sampling a continuous random process. To ensure that the proposed waveforms have Low Probability Intercept (LPI) characteristic, it is necessary to evaluate the Mutual Information Rate (MIR) i.e. the additional information that is added when one more sample is observed [10]. MIR is related to the concepts of joint and conditional entropy of a stochastic process [11]. Knowing MIR, a well-known criterion for measuring the randomness of a process is the Spectral Flatness Measure $(0<S F M \leq 1)$, which is defined as the ratio of the geometric mean to the arithmetic mean of the power spectrum [12]. If $S F M \rightarrow 1$ it corresponds to a random signal (no significant information can be obtained by looking at longer blocks of samples). When $S F M \rightarrow 0$ the signal is strongly structured and can be considered as a deterministic signal.

In this paper we analyze the information rate of pseudo noise waveforms with their randomness increasing.

This paper is organized as follows. Chapter II describes the mathematical definition of the main parameters introduced to evaluate the mutual information of a signal. Chapter III introduces the generation of pseudo noise waveforms, whose entropy, information and MIR will be evaluated in chapter IV. Chapter V reports final considerations, conclusions and future perspectives.

\section{MATHEMATICAL DEFINITION OF MIR}

Given $n$ real random variables $\left\{X_{1}, X_{2}, \ldots, X_{n}\right\}$ having marginal probability density function $f\left(x_{i}\right)$ for $i=1,2, \ldots, n$, and joint probability density function $f\left(x_{1}, x_{2}, \ldots, x_{n}\right)$, the marginal entropy for each $X_{i}$ is defined as:

$$
H\left(x_{i}\right)=E\left[-\log \left[f\left(x_{i}\right)\right]\right]=-\int f\left(x_{i}\right) \log \left[f\left(x_{i}\right)\right] d x_{i}
$$

In the following, the natural logarithm will be used (note that when dealing with discrete random variables the equivalent formulation with $\log _{2}$ is more widely used). The joint entropy is:

$$
\begin{aligned}
& H\left(x_{1}, x_{2}, \ldots, x_{n}\right)= \\
& =-\int_{\mathbb{R}^{n}} f\left(x_{1}, x_{2}, \ldots, x_{n}\right) \ln \left[f\left(x_{1}, x_{2}, \ldots, x_{n}\right)\right] d x_{1} \ldots d x_{n}
\end{aligned}
$$

If $\left\{X_{1}, X_{2}, \ldots, X_{n}\right\}$ are independent, i.e. $f\left(x_{1}, x_{2}, \ldots, x_{n}\right)=$ $f\left(x_{1}\right) \cdot f\left(x_{2}\right) \cdot \ldots \cdot f\left(x_{n}\right)$, from (2) the joint entropy becomes:

$$
H\left(x_{1}, x_{2}, \ldots, x_{n}\right)=H\left(x_{1}\right)+H\left(x_{2}\right)+\cdots+H\left(x_{n}\right)
$$

The mutual information, related to the $n$ random variables, is given by:

$$
\begin{aligned}
& I\left(x_{1}, x_{2}, \ldots, x_{n}\right)= \\
& \quad=\int_{\mathbb{R}^{n}} f\left(x_{1}, x_{2}, \ldots, x_{n}\right) \ln \left[\frac{f\left(x_{1}, x_{2}, \ldots, x_{n}\right)}{f\left(x_{1}\right) \cdot f\left(x_{2}\right) \cdot \ldots \cdot f\left(x_{n}\right)}\right] d x_{1} \ldots d x_{n}
\end{aligned}
$$

If $\left\{X_{1}, X_{2}, \ldots, X_{n}\right\}$ are independent then $I\left(x_{1}, x_{2}, \ldots, x_{n}\right)=$ 0 , otherwise:

$$
I\left(x_{1}, x_{2}, \ldots, x_{n}\right)=\sum_{i=1}^{n} H\left(x_{i}\right)-H\left(x_{1}, x_{2}, \ldots, x_{n}\right)
$$

Using the mutual information, the Marginal Information Redundancy (or Mutual Information Rate, MIR) $\rho_{n}$, is defined as:

$$
\rho_{n}=I\left(x_{1}, x_{2}, \ldots, x_{n}\right)-I\left(x_{1}, x_{2}, \ldots, x_{n-1}\right)
$$

The quantity $\rho_{n}$ measures the rate of growth of the common information as a function of the time. If $\left\{X_{1}, X_{2}, \ldots, X_{n}\right\}$ are independent, then $\rho_{n}=0$.

By (5) $\rho_{n}$ can be written as:

$$
\rho_{n}=H\left(x_{n}\right)-H\left(x_{1}, x_{2}, \ldots, x_{n}\right)+H\left(x_{1}, x_{2}, \ldots, x_{n-1}\right)
$$

Using the relation (chain rule) between the joint and the conditional densities for $\left\{X_{1}, X_{2}, \ldots, X_{n}\right\}: f\left(x_{1}, x_{2}, \ldots, x_{n}\right)=$ $f\left(x_{n} \mid x_{n-1}, \ldots, x_{1}\right) \cdot f\left(x_{n-1} \mid x_{n-2}, \ldots, x_{1}\right) \cdot \ldots \cdot f\left(x_{2} \mid x_{1}\right) \cdot f\left(x_{1}\right)$, the joint entropy can be written as a function of the conditional entropy:

$$
H\left(x_{1}, x_{2}, \ldots, x_{n}\right)=H\left(x_{n} \mid x_{n-1}, \ldots, x_{1}\right)+H\left(x_{n-1} \mid x_{n-2}, \ldots, x_{1}\right)+\ldots
$$$$
\ldots+H\left(x_{2} \mid x_{1}\right)+H\left(x_{1}\right)
$$

Therefore equation (7) becomes:

$$
\rho_{n}=H\left(x_{n}\right)-H\left(x_{n} \mid x_{1}, x_{2}, \ldots, x_{n-1}\right)
$$

The parameter $\rho_{n}$ represents the entropy of a single sample, $H\left(x_{n}\right)$, reduced by the knowledge of its past (conditional entropy). In the case of a Strictly Sense Stationary (SSS) process: $H\left(x_{n}\right)=H(x)$ independent of $n$, while the conditional entropy depends only on the vector size $n$ that contains the previous samples of the process. Introducing the entropy rate $H_{r}\left(x_{1}, x_{2}, \ldots, x_{n}\right)$, defined as the average uncertainty per sample in a block of $n$ consecutive samples [9]:

$$
H_{r}\left(x_{1}, x_{2}, \ldots, x_{n}\right)=\lim _{n \rightarrow \infty} \frac{1}{n} H\left(x_{1}, x_{2}, \ldots, x_{n}\right)
$$

if the limit exists. For a SSS process it can be demonstrated that the entropy rate equals its conditional entropy when $n \rightarrow \infty$, i.e. [11]:

$$
H_{r}\left(x_{1}, x_{2}, \ldots, x_{n}\right)=H\left(x_{n} \mid x_{1}, x_{2}, \ldots, x_{n-1}\right)
$$

Since the process is SSS, equation (10) can be rewritten as:

$$
\begin{aligned}
& H\left(x_{1}, x_{2}, \ldots, x_{n}\right)= \\
& =H(x)+\sum_{k=1}^{n-1} H\left(x_{k+1} \mid x_{k}, x_{k-1}, \ldots, x_{1}\right), \quad n \rightarrow \infty
\end{aligned}
$$

Dividing (12) by $n$, when $n \rightarrow+\infty$ the left term coincides with the entropy rate, while the right one, applying the Cesàro mean theorem, converges to the conditional entropy $H\left(x_{n} \mid x_{1}, x_{2}, \ldots, x_{n-1}\right)$. Summing up, for a SSS process, $\rho_{n}$ asymptotically shows the difference between the marginal entropy $H(x)$ of the process and its entropy rate $H_{r}\left(x_{1}, x_{2}, \ldots, x_{n}\right)$ :

$$
\rho_{n}=H(x)-H_{r}\left(x_{1}, x_{2}, \ldots, x_{n}\right), \quad n \rightarrow \infty
$$


We consider now the relevant case of Mutual Information Rate for a real SSS Gaussian process.

Assuming a SSS Gaussian, discrete-time process with power spectrum $S(\omega)$ where $\omega=2 \pi \frac{f}{F}$ ( $F$ is the sampling frequency), $\rho_{n}$ is evaluated by a separate estimation of the marginal entropy $H(x)$ and the entropy rate $H_{r}\left(x_{1}, x_{2}, \ldots, x_{n}\right)$. Each random variable $X_{i}=X\left(t_{i}\right)$, extracted from the process at the instant $t_{i}$, has a Gaussian density function with zero mean and variance $\sigma^{2}$. The marginal entropy (it is well known that this is maximum over all SSS random processes with different distributions) results [11]: $H(x)=\ln \left[\sqrt{2 \pi \sigma^{2} e}\right]$.

Replacing $\sigma^{2}$ with the average power of the process: $P=\frac{1}{2 \pi} \int_{-\pi}^{+\pi} S(\omega) d \omega$, the marginal entropy becomes:

$$
H(x)=\frac{1}{2} \ln \left[\frac{1}{2 \pi} \int_{-\pi}^{+\pi} S(\omega) d \omega\right]+\ln [\sqrt{2 \pi e}]
$$

The entropy rate $H_{r}\left(x_{1}, x_{2}, \ldots, x_{n}\right)$ of a SSS Gaussian process, also called Sinai-Kolmogorov Entropy, is [11]:

$$
H_{r}\left(x_{1}, x_{2}, \ldots, x_{n}\right)=\frac{1}{4 \pi} \int_{-\pi}^{+\pi} \ln [S(\omega)] d \omega+\ln (\sqrt{2 \pi e})
$$

Therefore:

$$
\rho_{n}=\frac{1}{2} \ln \left[\frac{1}{2 \pi} \int_{-\pi}^{+\pi} S(\omega) d \omega\right]-\frac{1}{4 \pi} \int_{-\pi}^{+\pi} \ln [S(\omega)] d \omega
$$

We observe that $\rho_{n}$ has the following properties [12]: (i) $\rho_{n} \geq 0$, it is due to the fact that $-\ln (\cdot)$ is a convex function, and then applying Jensen's inequality, i.e. $\ln \left[\int g(a) d a\right] \geq \int \ln [g(a)] d a$, the property is verified; (ii) $\rho_{n}=0$ if and only if $S(\omega)=$ cost., i.e. for a white process. From (16) we can introduce the Spectral Flatness Measure (SFM) [10]:

$$
S F M=\exp \left(-2 \rho_{n}\right)=\frac{\exp \left[\frac{1}{2 \pi} \int_{-\pi}^{+\pi} \ln [S(\omega)] d \omega\right]}{\frac{1}{2 \pi} \int_{-\pi}^{+\pi} S(\omega) d \omega}
$$

SFM is a well-known accepted method for evaluation of the "whiteness" (or "compressibility" in audio or imaging applications) of a signal. It can be shown that $0<S F M \leq 1$, which also follows from the non-negativity of $\rho_{n}$. Values of $S F M$ close to zero correspond to a structured (or non-random) signal. $S F M=1$ corresponds to a random, unpredictable signal. We can estimate $\rho_{n}$ inverting (17). The concepts mentioned above can be extended to complex processes as reported in [13] - [15].

\section{NOISY WAVEFORMS DESIGN METHODS}

In [16] has been developed a waveform whose ACF has very low sidelobes $(\sim-80 d B)$. This waveform is called Hybrid-NLFM because is achieved by a combination between a linear law and a tangential one.

Therefore it is well known that Hybrid-NLFM waveforms can provide good Peak Side Lobe Ratio (PSLR) of the autocorrelation even with a low compression ratio, and good cross correlation (orthogonality) between up and down signals with an increasing compression ratio. The drawback is their limited number (only two) and their LPI in a defense context. Now we introduce and compare two methods for generating noisy waveforms. The goal of these methods is good PSLR and orthogonality, with a large number of generated sequences, in addition to the LPI characteristics. We will show that a tradeoff between the different requirements is necessary. These two methods are a modified versions of the Advanced Pulse Compression Noise (APCN) radar waveforms introduced in [17], [18].

In the first method (named Hybrid-APCN, H-APCN for short) the waveform is obtained as:

$$
s_{H-A P C N}(t)=\alpha(t) \cdot e^{j k \cdot \varphi_{n o i s e}(t)} \cdot e^{j \varphi_{H N L F M}(t)}
$$

where: $\varphi_{\text {HNLFM }}(t)$ is the Hybrid-NLFM phase as defined in [16] (in [17] the phase follows a LFM law); $\alpha(t)$ is a rectangle of unit amplitude and duration $\mathrm{T}$ (the signals are hard limited in amplitude); $\varphi_{\text {noise }}(t)$ is a random phase with uniform distribution in $[0,2 \pi]$, whose related signal $e^{j k \varphi_{\text {noise }}(t)}$ has a spectrum equal to the deterministic one, added to the deterministic phase $\varphi_{H N L F M}(t)$; the parameter $0 \leq k \leq 1$ limits the variations of the random phase term, i.e. for example if $k=0.5$, then $k \cdot \varphi_{\text {noise }}(t) \in[0, \pi]$. For H-APCN process, as the noise increases, the deterministic phase $\varphi_{H N L F M}(t)$ remains unchanged and is always present.

In the second method, called Semi-Deterministic (HNLFM-SD, H-SD for short) because the deterministic phase does not remain the same, the waveform is:

$$
S_{H-S D}(t)=\alpha(t) \cdot e^{j \beta \cdot \varphi_{\text {noise }}(t)} e^{j(1-\beta) \cdot \varphi_{H N L F M}(t)}
$$

where the parameter $0 \leq \beta \leq 1$ limits the variations of $\beta \cdot \varphi_{\text {noise }}(t)$ and $(1-\beta) \cdot \varphi_{H N L F M}(t)$ : for $\beta=1$ the phase $\varphi_{\text {HNLFM }}(t)$ vanishes. The noise $e^{j \beta \varphi_{\text {noise }}(t)}$ has a spectrum equal to the deterministic one. Fig. 1 and 2 show the power spectrum evolution varying $k$ and $\beta$ for a bandwidth of 50 $\mathrm{MHz}$ and a compression ratio $\mathrm{BT}$ of 4096 respectively for $s_{H-A P C N}(t)$ (Fig. 1) and $s_{H-S D}(t)$ (Fig. 2) signals.

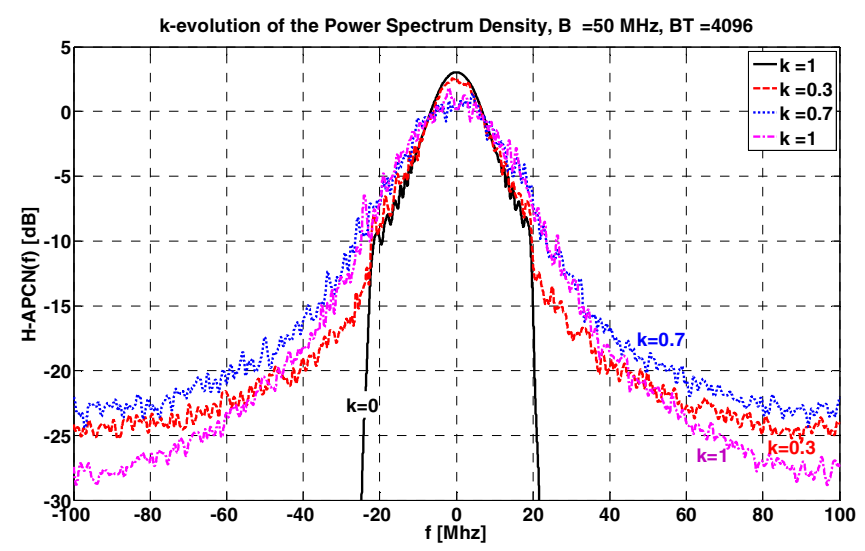

Figure 1. k-evolution of the Power Spectrum, B $=50 \mathrm{MHz}, \mathrm{BT}=4096$. 
Using the Short Time Fourier Transform, in Figures 3-6 are shown the spectrograms of the waveforms. Increasing $k$ or $\beta$ the random phase noise merges the deterministic phase. For the random phase limiter ( $k$ or $\beta$ ) greater than 0.7 the extraction of the instantaneous frequency law becomes very complicated. For Semi-Deterministic waveforms when $\beta \rightarrow 1$ the NLFM law degenerates into a straight line.

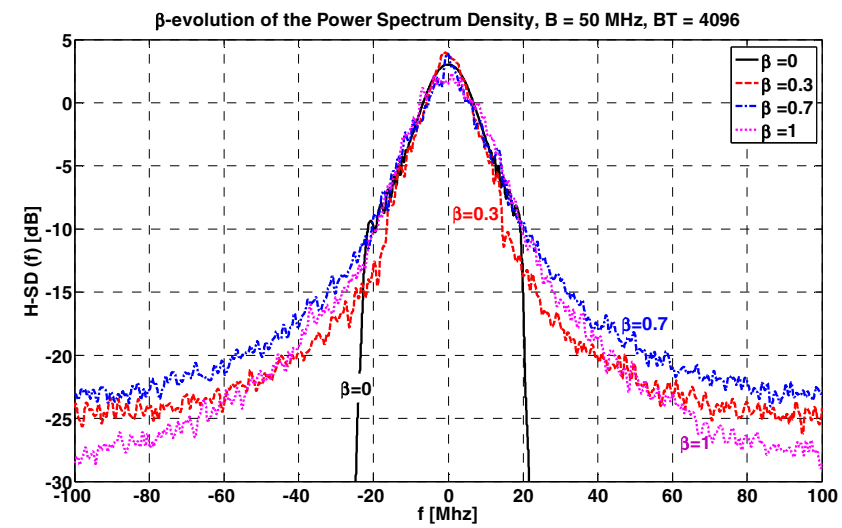

Figure 2. $\beta$-evolution of the Power Spectrum, B $=50 \mathrm{MHz}, \mathrm{BT}=4096$.
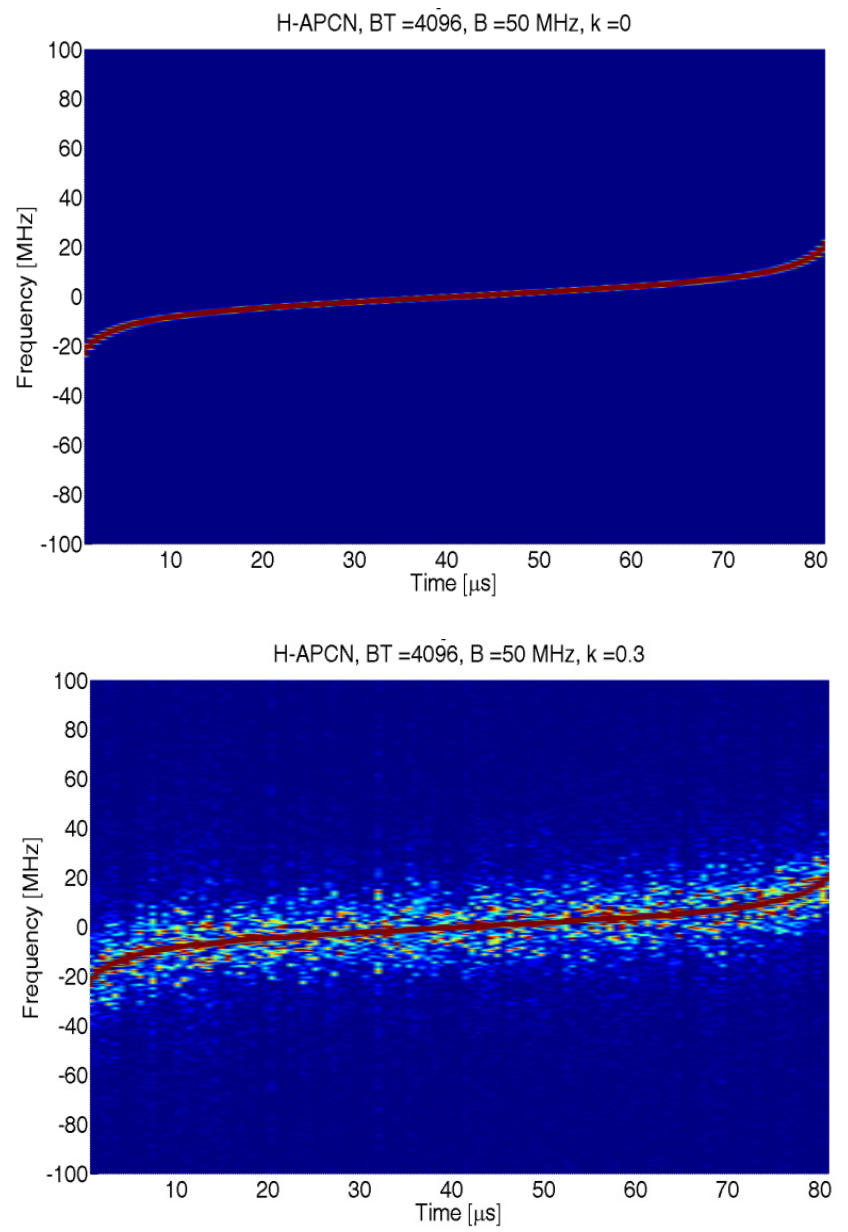

Figure 3. Spectrogram of the H-APCN waveform for $k=0,0.3$.
In Fig. 7 the mean PSLR (averaging 10 PSLR values) is shown versus the random parameters $k$ and $\beta$. Two waveforms were added; they are obtained substituting $\varphi_{H N L F M}(t)$ in (18) and (19) with $\varphi_{L F M}(t)$ for both APCN (LFM-APCN, L-APCN for short) and Semi-Deterministic (LFM-SD, L-SD for short) method. Fig. 7 shows that for $k, \beta \rightarrow 0$ the PSLR is $-43 \mathrm{~dB}$ and $-13 \mathrm{~dB}$, as expected for hard-limited NLFM and LFM respectively.

Increasing $k, \beta$ till 0.6 the PSLR regularly worsens for NLFM and improves for LFM with no evident difference between APCN and SD waveforms. For $k, \beta \geq 0.6$ the PSLR of $\mathrm{H}-\mathrm{APCN}$ and $\mathrm{H}-\mathrm{SD}$ signals remains quite constant $(\sim-25 d B)$ while the PSLR of L-SD signal worsens rapidly reaching $-15 d B$ which corresponds to a pure noise signal without deterministic phase (L-SD for $\beta=1$ ). For the case of L-APCN, even when $k \rightarrow 1$, the PSLR remains almost costant $(-28 \mathrm{~dB})$ being the spectrum of the L-APCN resulting by a combination between random phase and deterministic one which doesn't vanish unlike Semi-Deterministic case. With respect to the cross correlation, Fig. 8 shows, for all considered waveforms that when the random phase limiter increases, a regular improvement of the orthogonal property. However a good orthogonality $(-20 \mathrm{~dB})$ is reached only for $k, \beta>0.75$.
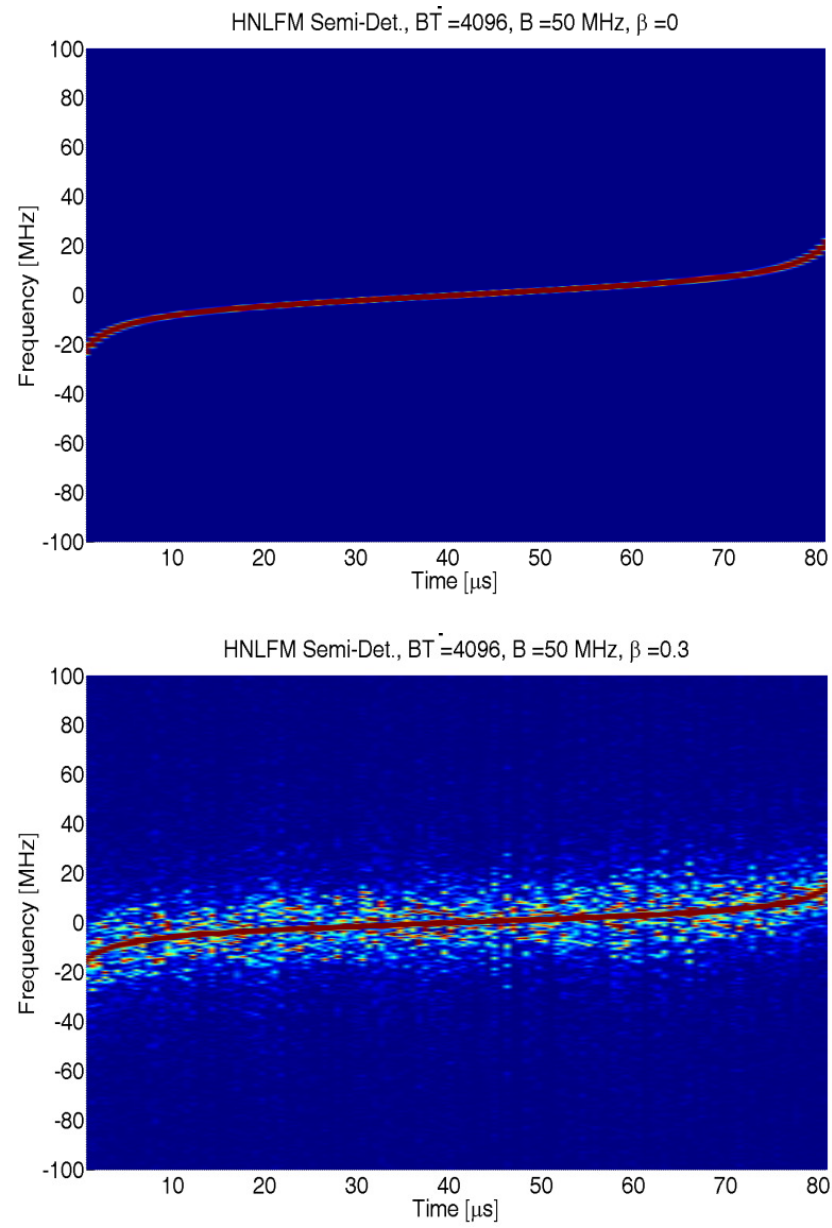

Figure 4. Spectrogram of the H-SD waveform for $\beta=0,0.3$. 

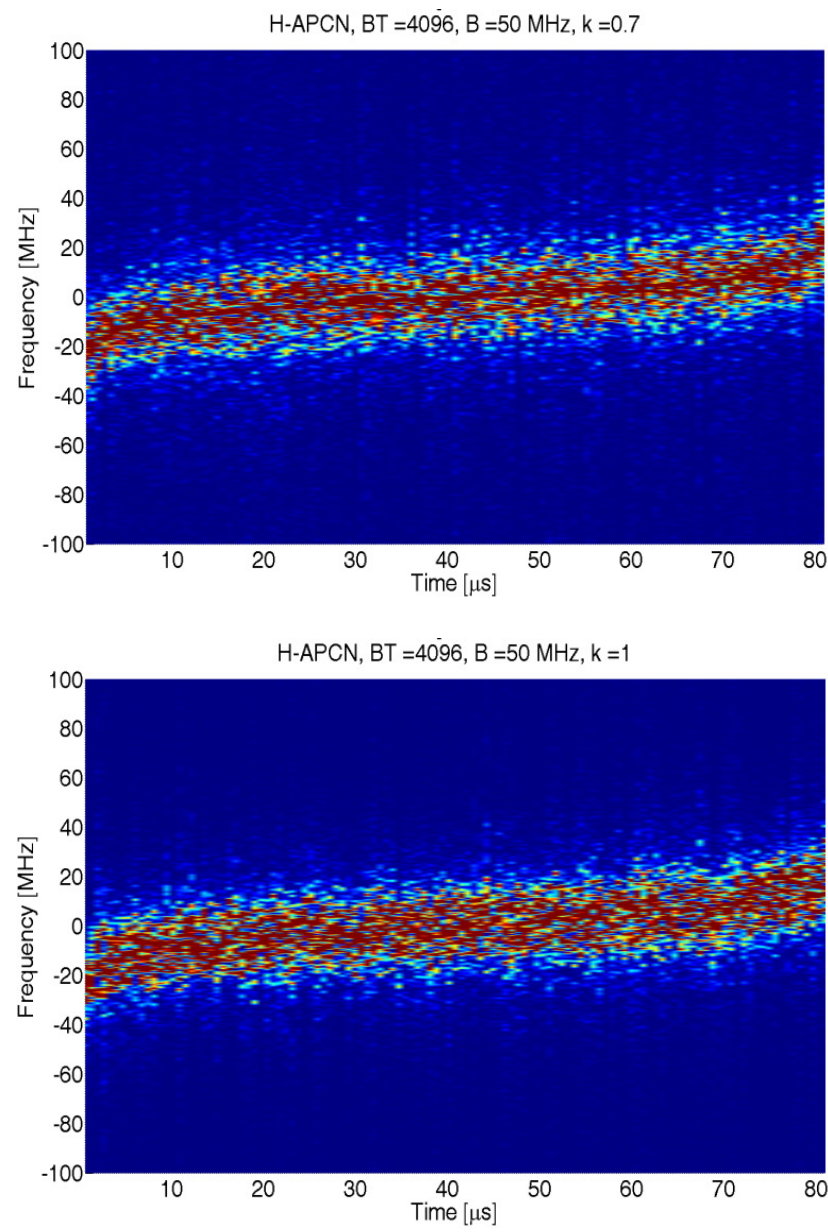

Figure 5. Spectrogram of the H-APCN waveform for $k=0.7,1$.

\section{MUlti INFORMATION RATE (MIR) Estimation}

Applying equation (16) MIR has been estimated for the waveforms previously introduced varying the random phase weight. Fig. 9 reports the results. Two signals are considered as reference: the first (continuous line) represents the case of only phase noise waveform, in which the phase is uniform between $(0,2 k \pi)$ being $0<k \leq 1$. Where $k \rightarrow 0$ the waveform is deterministic (i.e. structured signal) and MIR assumes very high value; the second (dot line) represents a band limited Gaussian white noise, whose MIR is always close to zero (maximum entropy signal due to pure Gaussian noise, with independent Gaussian I, Q components). For low values of $k, \beta$ the curves H-APCN and H-SD start from the same point because the signals are all-deterministic $(k, \beta=0)$.

For high values of $k, \beta(k, \beta \geq 0.8)$ the curves $\mathrm{H}$-APCN and H-SD don't reach the zero value (as expected because the added noise makes signal unpredictable) because the added noise doesn't change much the psd shape (see Fig. 1 and 2) which remains Gaussian-like, then the related MIR recognizes them as structured signals. In particular $\mathrm{H}$-SD power spectrum is narrower than H-APCN one (see Fig. 1 and 2 for $k, \beta \approx 1$ ),
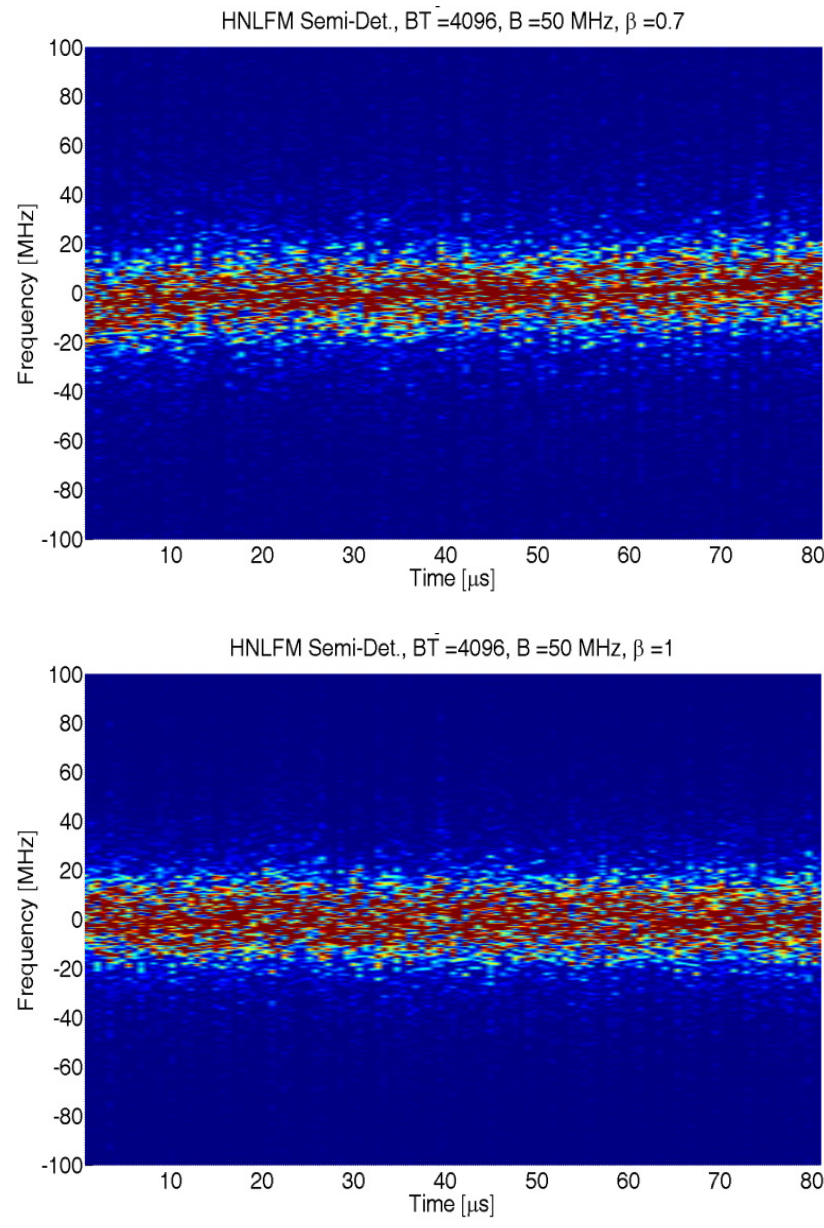

Figure 6. Spectrogram of the H-SD waveform for $\beta=0.7,1$.

then from point of view of the MIR the H-SD signal seem to be less "random" than H-APCN one.

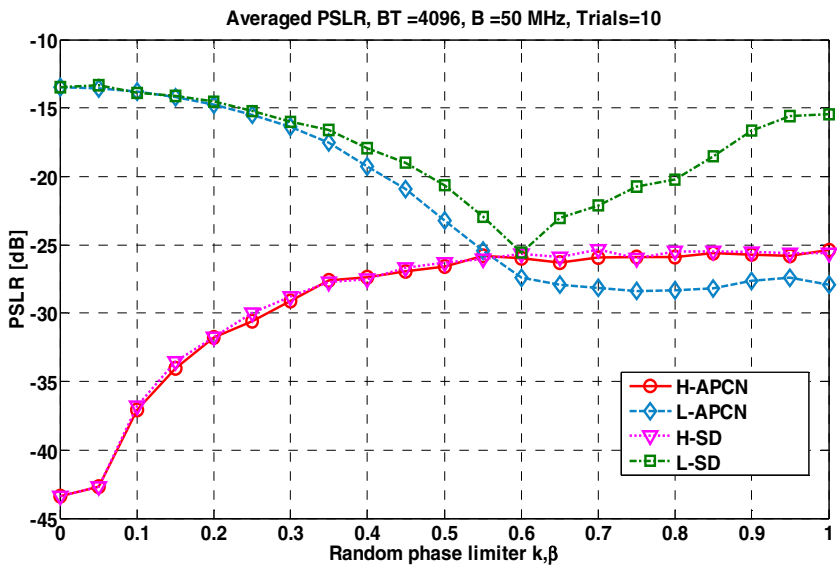

Figure 7. Average PSLR (10 trials) versus phase weight.

For L-APCN waveform MIR seems to be appropriate only if the random phase limiter is much greater than zero. 
L-APCN signal is a poor LPI waveform when $k$ is close to zero (pure LFM waveform), nevertheless from the point of view of the MIR seems to be very close to Gaussian noise, which represents instead the best LPI waveform. This incoherence is due to the fact that MIR takes into account only the total shape of the PSD, i.e. the total harmonic content, without considering the temporal evolution of the instantaneous frequency. That's why L-APCN and Gaussian noise have almost the same MIR close to zero.

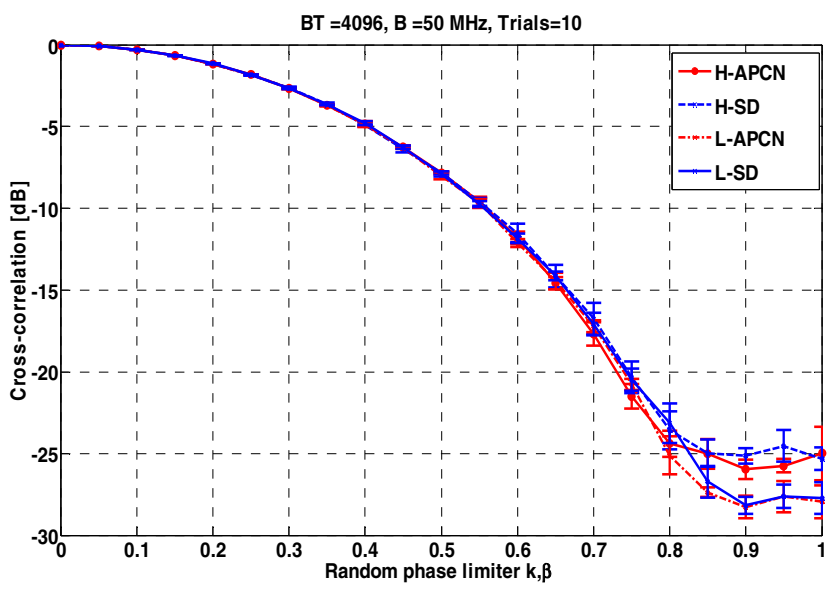

Figure 8. Cross-level average versus random phase weight.

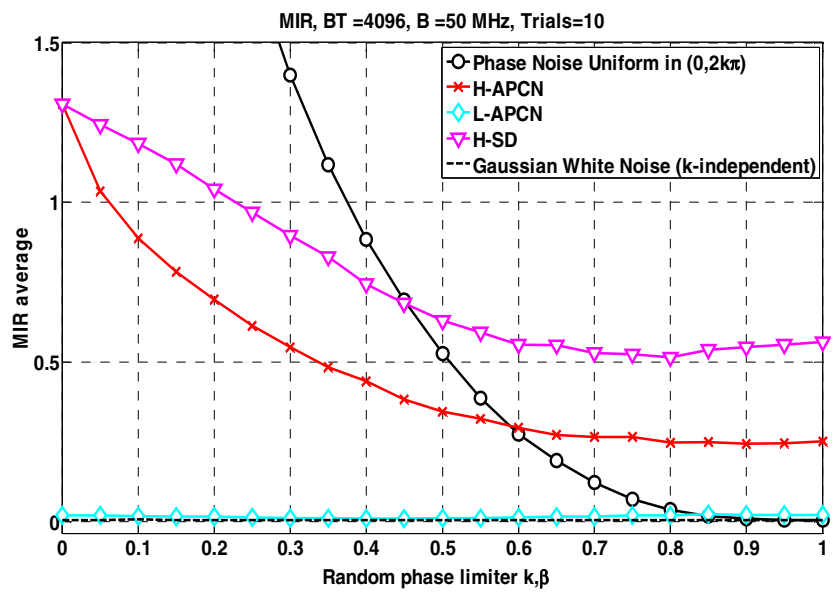

Figure 9. Multual Information Rate (MIR) versus Random Phase weight.

\section{Conclusion And Future Perspectives}

To summarize, if you are interested to orthogonal waveforms you must use high values of $k, \beta$. This leads to not optimized PSLR and good randomness (in the sense of the MIR). If you are interested to good PSLR waveforms, you must choose low $k, \beta$ values for H-APCN and H-SD signals, $\beta \approx 0.6$ for L-SD signal or $k>0.6$ for L-APCN one. Low $k, \beta$ values for H-APCN and H-SD signals lead to non- orthogonal waveforms and poor randomness in the signals (in the sense of the MIR).

For low values of phase weight, there isn't a big difference between H-APCN and H-SD signals, however in this case the $\mathrm{H}-\mathrm{APCN}$ signal is a bit more "random" (in the sense of the MIR) than H-SD one.

Future perspectives are related to prove on these waveforms the goodness of the recognition waveforms algorithms when the random phase weight increases.

\section{REFERENCES}

[1] Phillip E. Pace, Detecting and Classifying Low Probability of Intercept Radar, Second Edition, Artech House, 2009.

[2] K. Kulpa, Signal Processing in Noise Waveform Radar, Artech House, 2013, ISBN: 978-1-60807-661-1.

[3] K.A.Lukin, Noise Radar Technology, Telecommunications and Radio Engineering,Vol.55 (2001), No.12, pp 8-16.

[4] G. Galati, G. Pavan, On the Signal Design for Multifunction/Multiparameter Radar. Proc. of MRRS-Microwaves, Radar and Remote Sensing Symposium 2011. Kiev (Ukraine), 25-27 August 2011, pp. 2834, 2011.

[5] J. E. Gentle, Random Number Generators and Monte Carlo Methods, Second edition, Springer, 2003.

[6] Matlab Manual, www.mathworks.com.

[7] G. Galati, G. Pavan, Noise Radar Technology as an Interference Prevention Method. Journal of Electrical and Computer Engineering, vol. 2013, pp. 1-6, doi: 10.1155/2013/146986.

[8] G. Galati, G. Pavan, On the Design of Waveforms for Noise-MIMO Radar. Proc. of 3rd International Conference on Noise Radar Technology. Yalta (Ukraine), 27-29 september 2012, p. 1-7.

[9] A. Papoulis, Probability, Random Variables and Stochastic Processes, McGraw-Hill, 1984, pp. 531-535.

[10] S. Dubnov, Generalization of Spectral Flatness Measure for NonGaussian Linear Processes, IEEE Signal Processing Letters, vol. 11, n. 8, August 2004, pp. 698-701.

[11] A. Papoulis, Maximum Entropy and Spectral Estimation: A Review, IEEE Trans. On Acoustics, Speech and Dignal Processing, vol. ASSP29, n. 6, December 1981, pp. 1176-1186.

[12] K. Drouiche, A New Test for Whiteness, IEEE Trans. On Signal Processing, vol. 48, n. 7, July 2000, pp. 1864-1871.

[13] F. D. Neeser, J. L. Massey, Proper Complex Random Processes with Applications to Information Theory, IEEE Trans. on Information Theory, Vol. 39, N. 4, July 1993, pp. 1293-1302.

[14] Wei Xiong, Hualiang Li, Tülay Adali, Yi-Ou Li, V. D. Calhoun, On Entropy Rate for the Complex Domain and Its Application to i.i.d. Sampling, IEEE Trans on Signal Processing, Vol. 58, n. 4, April 2010, pp. $2409-2414$

[15] B. Picinbono, P. Bondon, Second-Order Statistics of Complex Signals, IEEE Trans. on Signal Processing, Vol. 45, N. 2, February 1997, pp $411-420$

[16] Zhiqiang Ge, Peikang Huang, Weining Lu, Matched NLFM Pulse Compression Method with Ultra-low Sidelobes, Proc. of the 5th European Radar Conference, October 2008, Amsterdam, pp. 92-95.

[17] M. A. Govoni, Range-Doppler Resolution of the Linear-FM Noise Radar Waveform, IEEE Trans. On Aerosp. And Electr. Systems Vol. 49, N. 1 January 2013, pp. 658-664.

[18] M. A. Govoni, Low Probability of Interception of an Advanced Noise Radar Waveform with Linear-FM, IEEE Trans. on Aerosp. and Electr. Systems Vol. 49, N. 2 April 2013, pp. 1351-1356. 